\title{
Automated Real-Time Atrial Fibrillation Detection on a Wearable Wireless Sensor Platform
}

\author{
Francisco Rincón, Paolo Roberto Grassi, Nadia Khaled, David Atienza and Donatella Sciuto
}

\begin{abstract}
This paper presents an automated real-time atrial fibrillation (AF) detection approach that relies on the observation of two characteristic irregularities of $\mathrm{AF}$ episodes in the electrocardiogram (ECG) signal. The results generated after the analysis of these irregularities are subsequently analyzed in real-time using a new fuzzy classifier. We have optimized this novel AF classification framework to require very limited processing, memory storage and energy resources, which makes it able to operate in real-time on a wearable wireless sensor platform. Moreover, our experimental results indicate that the proposed on-line approach shows a similar accuracy to stateof-the-art off-line AF detectors, achieving up to $96 \%$ sensitivity and $93 \%$ specificity. Finally, we present a detailed energy study of each component of the target wearable wireless sensor platform, while executing the automated AF detection approach in a real operating scenario, in order to evaluate the lifetime of the overall system. This study indicates that the lifetime of the platform is increased by using the proposed method to detect $\mathrm{AF}$ in real-time and diagnose the patient with respect to a streaming application that sends the raw signal to a central coordinator (e.g., smartphone or laptop) for its ulterior processing.
\end{abstract}

\section{INTRODUCTION}

Atrial fibrillation (AF) is the most common cardiac arrhythmia. It is associated with high risk of stroke, dementia, heart failure and death [1], and is predicted to be responsible for over 3 million hospitalizations by 2025 [2]. AF occurs when disorganized electrical signals cause the heart's atria to contract very fast and irregularly. This results in a desynchronization between the atria and the ventricles, which leads to an inefficient pump of blood. The early detection of $\mathrm{AF}$ is important to ensure dangerous (or even lethal) diseases to be identified and treated accordingly. However, it can be problematic since the AF can appear only during certain periods of time and may be asymptomatic. To overcome this problem, in this work we advocate the use of Wireless Body Sensor Network (WBSN) technologies, to enable continuous and advanced biomedical monitoring of the patients in real-time and therefore automated early diagnosis for better healthcare.

AF episodes can be identified by searching for anomalies in the electrocardiogram (ECG) signal, whose main waves (QRS complex, $\mathrm{P}$ and $\mathrm{T}$ waves) represent the different parts

This work was supported in part by the Spanish Government Research Grant TIN2008-00508.

F. Rincón is with the DACYA, Complutense University of Madrid, Madrid, Spain, and also with the ESL, Ecole Polytechnique Fédérale de Lausanne, Lausanne, Switzerland francisco.rincondfdi .ucm.es

P. R. Grassi and D. Sciuto are with the DEI, Politecnico di Milano, Milano, Italy $\{$ grassi, sciuto\}delet.polimi.it

N. Khaled is with the Bioanalytical Science Dept., Nestlé Research Center, Lausanne, Switzerland nadia.khaled@rdls. nestle.com

D. Atienza is with the ESL, Ecole Polytechnique Fédérale de Lausanne, Lausanne, Switzerland david.atienza@epfl.ch

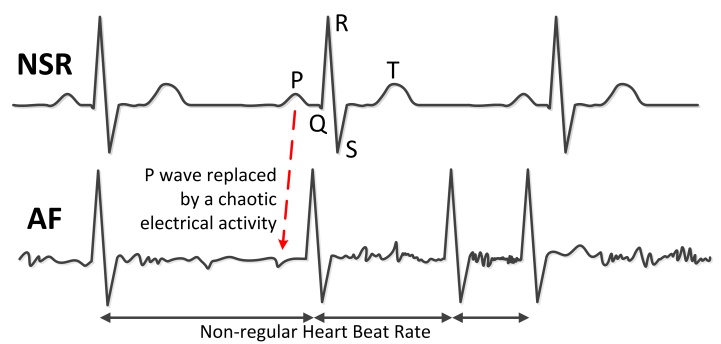

Fig. 1. ECG during normal sinus rhythm (above) and AF episode (below)

of the heart's cycle. The main characteristics of AF are an irregular heart beat rate (HBR), leading to a dramatic increase of the R-R interval variance, and the absence of the $\mathrm{P}$ wave, which is replaced by a sawtooth pattern. This is observed in Figure 1, which shows an ECG signal during normal sinus rhythm (NSR, above), where the different ECG waves can be clearly distinguished and the HBR is regular, and an AF episode (below), where the HBR is completely irregular and the $\mathrm{P}$ wave is replaced by a chaotic electrical activity between consecutive QRS complexes.

During the last two decades, several algorithms and techniques for the automatic detection and classification of AF episodes were presented. However, the implementation of such algorithms in a WBSN platform that can provide real-time monitoring and diagnosis is unheard of. Some of the algorithms we find in the literature focus on the analysis of the cardiac frequency to perform automatic AF classification [3], [4], [5]. However, these approaches require high computation and storage requirements, which do not make them applicable for WBSN platforms, since they work off-line using the whole ECG signal or very long windows of it. Other works, like [6], are based on the analysis of the $\mathrm{P}$ wave, taking also into account some statistical and electrophysiological properties of the signal, which suggests that combining various symptoms can be a key point for the implementation of accurate and efficient automatic detection algorithms. In this regard, [7] proposes the use of three different algorithms to detect AF episodes: $\mathrm{P}$ wave detection, heart rate analysis and atrial activity analysis. The outputs of these algorithms are subsequently combined using a neural network classifier. However, the atrial activity analysis involves quite complex frequency-domain calculations that exceed the capabilities of state-of-the-art WBSN nodes [8]. Also, the neural network classifier is very expensive in terms of computing power and memory resources to be implemented in such limited WBSN platforms.

This paper presents the development of a run-time approach for the automatic detection of AF episodes, based on 
the analysis of the HBR and the detection of the absence of the P wave in ECG signals. This approach has been optimized for its real-time execution on a low-power WBSN platform (i.e., Shimmer ${ }^{\mathrm{TM}}$ ), with very limited resources in terms of memory, processing capabilities and energy availability. Moreover, the proposed system achieves similar results to state-of-the-art off-line AF detection algorithms, reaching $96 \%$ sensitivity and $93 \%$ specificity, while providing continuous monitoring and diagnosis of the patient's heart activity.

The rest of the paper is organized as follows. Section II describes the proposed AF detection method, while Section III details the implementation and required optimizations for the Shimmer ${ }^{\mathrm{TM}}$ platform. The results of the proposed algorithm are discussed in Section IV, where also the energy consumption of the system in a realistic scenario is analyzed. Finally, Section V summarizes the conclusions of this work.

\section{ATRIAL FIBRILLATION DETECTION APPROACH}

As aforementioned, the AF detection approach proposed in this work relies on two algorithms that perform, for each heart beat, an analysis of the HBR and the detection of the absence of the $\mathrm{P}$ wave, respectively. Then, the outputs of both algorithms are combined using fuzzy logic to classify the heart beat under analysis as AF or normal. The scheme of the proposed technique, is similar to the one described in [7], but avoids the complex atrial activity analysis and replaces the neural network classifier by a much simpler, but yet highly accurate, fuzzy classifier. These changes allow the algorithm to meet the limited resources of the Shimmer ${ }^{\mathrm{TM}}$, especially in terms of memory and processing power. This section provides a detailed description of these algorithms as well as our new fuzzy classifier.

\section{A. Heart Rate Analysis}

Heart rate analysis is based on the $R$ - $R$ interval variance, which is higher during $\mathrm{AF}$ episodes due to the irregular activity of the heart. To detect the most significant peaks of the ECG signal (i.e., the R peaks), we use as starting point the algorithm presented in [9], which performs waveletbased single-lead detection of the QRS complex, $\mathrm{P}$ and $\mathrm{T}$ waves, so-called ECG delineation. Then, when the R peaks are detected, we calculate the time difference between two consecutive peaks (R-R intervals). Next, we normalize the $\mathrm{R}-\mathrm{R}$ intervals according to the following equation:

$$
R R_{\text {norm }}=\frac{R R}{\overline{R R}} * 100 ;
$$

where $R R$ is the current $\mathrm{R}-\mathrm{R}$ interval, $\overline{R R}=\frac{2}{3} \bar{R} R+\frac{1}{3} R \bar{R}^{\prime}$ and $R \bar{R}^{\prime}$ is the average of the R-R intervals in a 10-second window centered in the current beat. This formula is inspired by the feature normalization proposed in [10], and updates $\overline{R R}$ with local information about the current $\mathrm{R}-\mathrm{R}$ interval, compensating for different patient resting HBR. The variance of $R R_{\text {norm }}$ is then computed over a 10 -second sliding window.

At this point, [5] applies hard thresholding to the variance in order to compute an initial AF detection. This initial classification is then smoothed to eliminate spurious errors using a majority voting scheme over a 600-beat window. Instead, in order to reduce the computational complexity and storage requirements to be able to run in real-time in WBSN nodes, we provide the variance to our newly developed fuzzy classifier, that will interpret this data accordingly. Moreover, this new approach we propose enables a much faster diagnosis, with an average delay to classify a beat of only 5 seconds, since it uses a 10 -second sliding window centered in the current beat, while the average delay of the method proposed in [5] is 300 heart beats, which means 225 seconds for a typical HBR of 80 beats per minute.

\section{B. $P$ wave detection}

We perform the $\mathrm{P}$ wave detection by comparing the candidate $\mathrm{P}$ wave automatically obtained by the previously mentioned wavelet-based delineation algorithm with a model of the $\mathrm{P}$ wave, inspired on the approach proposed by [7]. However, in our case this model was built by averaging all the annotated P waves found in the QT database [11]. Then, for each beat, we calculate the correlation coefficient between the $\mathrm{P}$ wave detected by the delineation algorithm and our $\mathrm{P}$ wave model. This correlation value is then given as an input to our fuzzy classifier.

\section{Fuzzy Classifier}

To combine the outcome of both sub-algorithms, we have developed a specific fuzzy classifier for AF detection. Thanks to its low complexity, it can be implemented to fit the limited resources of state-of-the-art WBSN platforms, such as the Shimmer ${ }^{\mathrm{TM}}$ node.

The classifier takes the output of the sub-algorithms and provides the outcome of classification process (NSR or AF). Using trapezoidal membership functions, they translate the raw output of the two sub-algorithms into a fuzzy value that is subsequently used by fuzzy rules to detect and classify AF episodes. Each membership function is characterized by four parameters, which define the borders of fuzzy values in the process of fuzzification: A (for HBR analysis) and $\mathbf{B}$ (for $\mathrm{P}$ wave correlation). In this specific problem, each fuzzy variable can be represented with three fuzzy values: $\mathbf{A F}$ (AF episode detected), NO AF (normal sinus rhythm) and UNK (it is not clear if an AF episode is happening or not). In the latter case, additional information is required (i.e. the output of the other membership function). To reliably detect AF episodes, and after a careful experimental exploration, we have set these parameters to the values indicated in Table I, which provide the best results. The developed membership functions are depicted in Figure 2.

To detect AF episodes, our proposed classifier combines fuzzy values using the rules presented in Table II, which

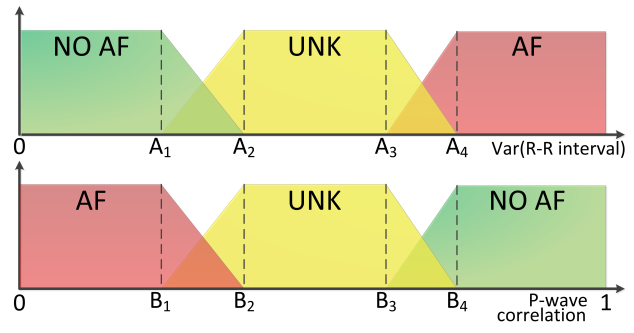

Fig. 2. Membership functions of our fuzzy classifier. 
TABLE I

CONFIGURATION OF THE PARAMETERS OF OUR FUZZY CLASSIFIER

\begin{tabular}{c|c|c|c||c|c|c|c}
\hline$A_{1}$ & $A_{2}$ & $A_{3}$ & $A_{4}$ & $B_{1}$ & $B_{2}$ & $B_{3}$ & $B_{4}$ \\
\hline 150 & 200 & 250 & 300 & 0.5 & 0.6 & 0.7 & 0.8 \\
\hline
\end{tabular}

TABLE II

FUZZY RULES USED FOR AF CLASSIFICATION

\begin{tabular}{c|c|c|c}
\hline Var(R-R interval) & P wave correlation & output value & output weight \\
\hline $\mathrm{AF}$ & $\mathrm{AF}$ & $\mathrm{AF}$ & 1 \\
\hline $\mathrm{AF}$ & - & $\mathrm{AF}$ & 0.5 \\
\hline- & $\mathrm{AF}$ & $\mathrm{AF}$ & 0.2 \\
\hline $\mathrm{AF}$ & $\mathrm{UNK}$ & $\mathrm{AF}$ & 0.8 \\
\hline $\mathrm{UNK}$ & $\mathrm{AF}$ & $\mathrm{AF}$ & 0.4 \\
\hline $\mathrm{NO} \mathrm{AF}$ & $\mathrm{UNK}$ & $\mathrm{NO} \mathrm{AF}$ & 1 \\
\hline $\mathrm{UNK}$ & $\mathrm{NO} \mathrm{AF}$ & $\mathrm{NO} \mathrm{AF}$ & 0.5 \\
\hline $\mathrm{NO} \mathrm{AF}$ & - & $\mathrm{NO} A F$ & 0.9 \\
\hline- & $\mathrm{NO} \mathrm{AF}$ & $\mathrm{NO} \mathrm{AF}$ & 0.5 \\
\hline $\mathrm{NO} \mathrm{AF}$ & $\mathrm{NO} \mathrm{AF}$ & $\mathrm{NO} \mathrm{AF}$ & 1
\end{tabular}

are weighted according to the accuracy and the correlation between symptoms and correct AF classification. In addition, Table I shows the configuration of the parameters of the fuzzy classifier, which was set to obtain the best results on all the ECG records of the database used to validate the proposed approach, each of which belongs to a different patient. However, since the ECG signals differ from patient to patient and depend on physiological aspects like heart size or chest structure, the parameters of the classifier can be tuned further by training to improve classification accuracy on a specific patient. In fact, our results indicate that a personalized configuration for each patient always enhances the accuracy of the AF detection, achieving up to $100 \%$ sensitivity and specificity for several signals of the used database. This training can be done using small excerpts of available ECG traces of each patient that have been previously annotated by the cardiologist. Moreover, training not only improves classification itself, but can be used to improve single-algorithm accuracy.

\section{REAL-TIME EMBEDDED AF DETECTION}

Our target WBSN node is the Shimmer ${ }^{\mathrm{TM}}$ [8], a small, lowpower commercial wireless sensor platform for noninvasive biomedical research. The Shimmer ${ }^{\mathrm{TM}}$ node is equipped with an ultra-low-power 16-bit microcontroller (TI MSP430), that runs at a maximum clock frequency of $8 \mathrm{MHz}$ and includes $10 \mathrm{~KB}$ of RAM and $48 \mathrm{~KB}$ of Flash, as well as some peripherals such as an 8-channel analog to digital (A/D) converter and a direct memory access unit (DMA). This platform has also two radios (Bluetooth and IEEE 802.15.4-compliant), a 3-axis accelerometer and an expansion port to connect a daughter board that can include additional sensors (ECG, electromyogram, galvanic skin response, etc.). For this work we have used the IEEE 802.15.4-compliant radio available in the Shimmer ${ }^{\mathrm{TM}}$, and a board that is capable of acquiring 3-lead ECGs.

With respect to the OS software, we ported FreeRTOS [12], which is a portable, open source, and hard real-time mini kernel with support for the microcontroller and the IEEE 802.15.4-compliant radio chip used by Shimmer ${ }^{\mathrm{TM}}$. Finally, we have used the open source GCC 3.2.3 for MSP430 to generate the binaries of our AF detection approach.
Regarding the adaptations of the proposed AF detection approach for the limited resources of the Shimmer ${ }^{\mathrm{TM}}$ platform, one of the most important parts in the design is the module that performs the detection of the $\mathrm{R}$ peaks, since a precise computation of the $\mathrm{R}-\mathrm{R}$ intervals relies on an accurate detection of the $\mathrm{R}$ peaks. The algorithm that performs this duty is the wavelet-based delineator presented in [9]. We mainly selected this algorithm for its low memory usage (it only needs $6.5 \mathrm{~KB}$ of RAM) and its low computation requirements (in only requires a $4 \%$ duty cycle). This algorithm also performs the delineation of the $\mathrm{P}$ and $\mathrm{T}$ waves, and therefore we can reuse the delineation of the $\mathrm{P}$ wave as an input for the module of the AF detection algorithm that performs the analysis of the absence of the $\mathrm{P}$ wave.

Moreover, the proposed algorithm has been optimized to reduce the memory usage and execution time, and therefore achieve a long lifetime of the platform while performing an accurate AF detection in real-time. After these optimizations, the memory used by our complete AF detection approach algorithm is $8.4 \mathrm{~KB}$ of RAM, which means that all the required processing for ECG classification and $\mathrm{AF}$ detection after the delineation algorithm only needs $1.9 \mathrm{~KB}$ of RAM. This low memory usage is mainly due to the use of our new fuzzy classifier, that requires very few resources while achieving a highly accurate classification. Overall, the duty cycle of our complete real-time AF detection approach is only $7.73 \%$, enabling the MSP430 to go to sleep mode during the remaining $92.27 \%$ of the time and therefore resulting in an extended lifetime of the Shimmer ${ }^{\mathrm{TM}}$ (cf. Section IV).

\section{EXPERIMENTAL RESULTS}

To evaluate the performance of the proposed algorithm, we analyze its detection accuracy and compare it to other state-of-the-art algorithms. Finally, an energy consumption study is performed to evaluate the lifetime of the WBSN node in real operation scenarios.

\section{A. AF Detection Results}

To assess the AF detection accuracy of the proposed algorithm, we have used the MIT-BIH AF database (AFDB) [10], which is composed of 23 10-hour ECG recordings sampled at $250 \mathrm{~Hz}$, with manual annotations of the AF episodes that appear in the signals. To this end, a beat-to-beat comparison between the results obtained by the algorithm and the manual annotations of the database is performed. Then, we calculate the sensitivity $(S e)$ and specificity $(S p)$, which are defined as:

$$
S e=\frac{T P}{T P+F N}, \quad S p=\frac{T N}{T N+F P}
$$

where $T P$ is the number of true positive detections, $F N$ is the number of false negative detections, $T N$ is the number of true negative detections and $F P$ is the number of false positive detections.

The results obtained after running the algorithm over the considered database show that the proposed system achieves 96\% sensitivity and 93\% specificity. These results are comparable to state-of-the-art off-line AF detection algorithms, even outperforming several of them as Table III shows, while the proposed AF algorithm is able to operate on-line in 
TABLE III

PERFORMANCE COMPARISON BETWEEN THE PROPOSED AF DETECTION APPROACH AND STATE-OF-THE-ART OFF-LINE ALGORITHMS. *THESE VALUES CORRESPOND TO POSITIVE PREDICTIVITY $\left(P+=\frac{T P}{T P+F P}\right)$

\begin{tabular}{c|c|c}
\hline Algorithm & $S e(\%)$ & $S p(\%)$ \\
\hline This work & 96 & 93 \\
\hline Couceiro et al. [7] & 93.8 & 96.09 \\
\hline Moody et al. [10] & 93.58 & $85.92^{*}$ \\
\hline Cerutti et al. [3] & 93.3 & $94 *$ \\
\hline Tateno et al. [4] & 93.2 & 96.7 \\
\hline Logan et al. [5] & 96 & 89 \\
\hline
\end{tabular}

TABLE IV

NODE LIFETIME FOR STREAMING AND AF DETECTION

\begin{tabular}{l|l|l}
\hline & ECG streaming & AF detection \\
\hline Code execution time $(\%)$ & 0 & 7.73 \\
\hline Packet ready every... $(\mathrm{ms})$ & 304 & 21000 \\
\hline Beacon interval $(\mathrm{ms})$ & 983 & 62915 \\
\hline Energy consumption $(\mathrm{mJ})$ & 7.70 & 7.41 \\
\hline Lifetime $(\mathrm{h})$ & 134.6 & 139.9 \\
\hline
\end{tabular}

WBSN nodes and provides real-time AF monitoring and detection. In order to test the generalization capability of the system, another experiment was performed, using 8 signals of the database for training and the remaining 15 for testing, obtaining $96 \%$ sensitivity and $91 \%$ specificity for the training set and $95 \%$ sensitivity and $91 \%$ specificity for the test set.

\section{B. Energy consumption comparison vs. ECG streaming}

This section provides an energy consumption analysis of the proposed system as well as a comparison with ECG data streaming, to evaluate how onboard processing affects the total energy consumption of the sensor node, using the power consumption characterization of the Shimmer ${ }^{\mathrm{TM}}$ platform proposed in [13]. In this work, we have used a reduced version of the beacon-enabled mode of the IEEE 802.15.4 protocol using guaranteed time slots (GTS) over a 8-node star network. The nodes can transmit up to four complete packets during their time slot. During the rest of the time the radio of the nodes is in low-power mode.

According to this power characterization, Table IV shows the energy consumption of the Shimmer ${ }^{\mathrm{TM}}$ running an ECG streaming application, that sends the raw ECG data sampled at $250 \mathrm{~Hz}$ to the WBSN coordinator, as well as the proposed AF detection algorithm. The first row shows the code execution in percentage of ECG signal acquisition time, while the second row shows the time interval between the generation of two consecutive packets. In the case of the streaming application, since the ADC offers a 12-bit resolution and the maximum payload of the packet is 114 bytes, we conclude that each packet can contain 76 ECG samples, and therefore the time interval between two consecutive packets is $304 \mathrm{~ms}$. In the case of the AF detection algorithm, only the results of the classification are sent to the WBSN coordinator, which means that, for each beat, the node sends the time where the beat was detected (31 bits) and the result of the classification ( 1 bit: $A F$ or normal). Therefore a packet can store 28 heart beats. Assuming a typical HBR of 80 beats per minute, a packet is generated every $21 \mathrm{~s}$. The third row shows the beacon interval, that is adjusted taking into account the maximum throughput required by the algorithm. The fourth row shows the energy consumption of the considered sensor platform per second. Finally, the last row indicates the total lifetime of the node by considering the energy supply of the Shimmer ${ }^{\mathrm{TM}}$, which has a $280 \mathrm{mAh}$ Li-ion battery at $3.7 \mathrm{~V}$. From Table IV we can conclude that although advanced processing is performed in the Shimmer ${ }^{\mathrm{TM}}$ WBSN node and therefore the energy consumption of the microcontroller increases, the total lifetime of the WBSN node including the real-time AF detection algorithm is a $4 \%$ higher than using a baseline ECG raw transmission, due to the significant reduction in the amount of information that is transmitted to the WBSN coordinator.

\section{CONCLUSIONS}

This paper has demonstrated the feasibility of the real-time implementation of AF detection on wearable wireless sensor platforms, based on the detection of two ECG characteristic features of this kind of episodes, namely the R-R interval irregularity and the absence of the $\mathrm{P}$ wave. This work has shown the importance of a sensible design and optimization of the AF detection approach for the limited processing and storage capabilities of the target WBSN platforms. As a result, the performance results of our complete real-time $\mathrm{AF}$ detection system are similar to state-of-the-art off-line AF detectors, achieving $96 \%$ sensitivity and $93 \%$ specificity for the AFDB. Finally, this work has presented an energy evaluation of the algorithm running on the Shimmer ${ }^{\mathrm{TM}}$ platform, which shows that although advanced signal processing is performed in the sensor node and thus the energy consumption of the microcontroller is higher, the lifetime of the overall system is increased by $4 \%$ with respect to streaming, since the amount of information that needs to be sent to the WBSN coordinator is dramatically reduced.

\section{REFERENCES}

[1] E. J. Benjamin et al. Prevention of atrial fibrillation: report from a national heart, lung, and blood institute workshop. In Circulation, vol. 119, pp. 606-618, 2009

[2] W. A. Wattingney and J. B. Croft. Atrial fibrillation hospitalizations triple since 1985 , will continue to climb. Available: http://www.charitywire.com/charity8/04025.html, 2003.

[3] S. Cerutti et al. Analysis of the dynamics of RR interval series for the detection of atrial fibrillation episodes. In Comp. in Cardiology, pp. 77-80, 1997.

[4] K. Tateno and L. Glass. A method for detection of atrial fibrillation using RR intervals. In Comp. in Cardiology, pp. 391-394, 2000.

[5] B. Logan et al. Robust detection of atrial fibrillation for a long term telemonitoring system. In Comp. in Cardiology, pp. 619-622, 2005.

[6] L. Clavier et al. Automatic P-wave analysis of patients prone to atrial fibrillation. Med. \& Biolog. Eng. \& Comp., 40(1):63-71, 2002.

[7] R. Couceiro et al. Detection of atrial fibrillation using model-based ECG analysis. In Int. Conf. on Pattern Recognition, pp. 1-5, 2008

[8] A. Burns et al. SHIMMER a wireless sensor platform for noninvasive biomedical research. IEEE Sensors, 10(9):1527-1534, 2010.

[9] N. Boichat et al. Wavelet-Based ECG Delineation on a Wearable Embedded Sensor Platform In IEEE International Workshop on Body Sensor Networks (BSN). 2009.

[10] G. B. Moody et al. A new method for detecting atrial fibrillation using R-R intervals. In Comp. in Cardiology, vol. 10, pp. 227-230, 1983.

[11] P. Laguna et al. A database for evaluation of algorithms for measurement of QT and other waveform intervals in the ECG. In Comp. in Cardiology, vol.24, pp. 673-676, 1997.

[12] Freertos real-time operating system. Available: http://www.freertos.org/.

[13] F. Rincón et al. Development and Evaluation of Multi-Lead WaveletBased ECG Delineation Algorithms for Embedded Wireless Sensor Nodes. In IEEE Transactions on Transactions on Information Technology in BioMedicine (TITB), vol.15, pp. 854-865. 